\title{
Lorlatinib-induced visual and auditory hallucinations: a case report
}

\author{
jun hakamata ${ }^{1}$, Hideo Nakada ${ }^{2}$, Hiroshi Muramatsu ${ }^{1}$, Keita Masuzawa ${ }^{3}$, Hideki Terai ${ }^{1}$, \\ Shinnosuke Ikemura ${ }^{3}$, Koichi Fukunaga ${ }^{3}$, and Tohru Aomori ${ }^{2}$ \\ ${ }^{1}$ Keio University Hospital \\ ${ }^{2}$ Keio University Faculty of Pharmacy Graduate School of Pharmacy \\ ${ }^{3}$ Keio University School of Medicine Graduate School of Medicine
}

November 12, 2020

\begin{abstract}
Lorlatinib is an anaplastic lymphoma kinase tyrosine kinase inhibitor for the treatment of non-small-cell lung cancer. We report a case in which visual and auditory hallucinations developed while receiving lorlatinib.

Lorlatinib-induced visual and auditory hallucinations: a case report

Jun Hakamata ${ }^{1}$, Hideo Nakata ${ }^{2}$, Hiroshi Muramatsu ${ }^{1}$, Keita Masuzawa ${ }^{3,4}$, Hideki Terai ${ }^{5}$, Shinnosuke Ikemura $^{3,6}$, Koichi Fukunaga ${ }^{3}$, Tohru Aomori ${ }^{1,2}$

${ }^{1}$ Department of Pharmacy, Keio University Hospital, Tokyo, Japan

${ }^{2}$ Division of Hospital Pharmacy Science, Faculty of Pharmacy, Keio University, Tokyo, Japan

${ }^{3}$ Division of Pulmonary Medicine, Department of Medicine, Keio University School of Medicine, Tokyo, Japan

${ }^{4}$ Division of Pulmonary Medicine, Department of Medicine, Kitasato University Kitasato Institute Hospital, Tokyo, Japan

${ }^{5}$ Keio University Hospital Clinical and Translational Research Center, Tokyo, Japan

${ }^{6}$ Department of Cancer center, Keio University Hospital, Tokyo, Japan

Correspondence
\end{abstract}

Jun Hakamata, Department of Pharmacy, Keio University Hospital 35 Shinanomachi, Shinjuku-ku, Tokyo 160-8582, Japan

Phone \& Fax: +81-3-3353-1211 E-mail: jun.hkmt@adst.keio.ac.jp

What is known and objective:

Lorlatinib is an anaplastic lymphoma kinase tyrosine kinase inhibitor for the treatment of non-small-cell lung cancer. We report a case in which visual and auditory hallucinations developed while receiving lorlatinib.

Case summary:

A 72-year-old Japanese man developed grade 2-3 visual and auditory hallucinations while taking lorlatinib. The hallucinations disappeared after discontinuing the lorlatinib, but grade 1 hallucinations developed after readministration. The hallucinations were a suspected adverse drug reaction. Lorlatinib was discontinued due to worsening general condition but the auditory hallucinations did not improve. 
What is new and conclusion:

This case is significant because lorlatinib is still infrequently administered, having been approved as recently as November 2018 in Japan.

Keyword: lorlatinib, hallucinations, non-small-cell lung cancer, Japanese patient

Key Clinical Message:Lorlatinib can cause visual and auditory hallucinations. And, it is necessary to keep in mind that hallucinations can persist even after discontinuation in patients who develop hallucinations while receiving lorlatinib.

What is known and objective

Around $3 \%-5 \%$ of patients with non-small-cell lung cancer (NSCLC) are positive for anaplastic lymphoma kinase $(\mathrm{ALK})$ and they are susceptible to treatment with an ALK tyrosine kinase inhibitor (ALK-TKI). ${ }^{1-3}$ In Japan, crizotinib, alectinib, and ceritinib are used in the primary treatment of ALK-positive NSCLC, but relapse can still occur due to secondary mutations in the ALK domain and exacerbations in the central nervous system (CNS). ${ }^{4,5}$

Lorlatinib, a third-generation ALK-TKI targeting ALK and c-ros oncogene 1 (ROS1) kinases, is expected to be effective in patients with ALK-TKI resistance. ${ }^{6}$ It is designed to penetrate the blood-brain barrier and it has efficacy against known ALK resistance as well as brain metastasis. ${ }^{7,8}$

It was approved in Japan in November 2018 and therefore has not been administered in a large number of cases as yet.

CNS disorders have been reported as characteristic adverse events associated with lorlatinib. A safety analysis of a phase I/II trial of lorlatinib $(n=295)$ recorded the following effects as CNS disorders: cognitive effects in $23.1 \%$ of patients (1.7\% grade $3-4)$; mood effects in $21.0 \%$ (1.4\% grade $3-4)$; and speech effects in $14.2 \%$ (0.3\% grade $3-4) .{ }^{7}$ However, there have been no detailed reports on hallucinations caused by lorlatinib among patients with the CNS disorders.

Here, we report in detail a case in which lorlatinib administration was associated with visual and auditory hallucinations, with the auditory hallucinations persisting after lorlatinib was discontinued.

Case summary

The patient was a 72-year-old man (height, $166.3 \mathrm{~cm}$; weight, $58.6 \mathrm{~kg}$ ) who was admitted with right heart failure. He had a history of cerebral infarction, type 2 diabetes, hypertension, non-sustained ventricular tachycardia, and constrictive pericarditis and no history of allergic reaction. He was receiving alectinib for ALK-positive NSCLC. On admission, computed tomography showed ground-glass opacity in the lungs, so the alectinib was discontinued due to suspected drug-induced lung injury and steroids were started. After the lung injury showed improvement, alectinib was switched to lorlatinib $100 \mathrm{mg} / \mathrm{day}$. At the time of switching to lorlatinib, both renal and liver function was normal. On the day 4 after lorlatinib was initiated, he experienced auditory hallucinations, followed by visual hallucinations 3 days later. The hallucinations were diagnosed as grade 2-3 CNS disorders. He reported hearing music playing outside the window or close to him, loudly or faintly, and involving familiar and unfamiliar tunes. In particular, he said the music was faint when talking with people. The visual hallucinations ranged from mild, such as curtains approaching him up close and stuffed animals emerging from the ceiling, to severe, including his bed turning into an airplane or train and seeing a variety of magnificent views and cityscapes from the window. Concomitant medications were the same as before admission and before the visual and auditory hallucinations developed: vonoprazan fumarate $(10 \mathrm{mg} /$ day), a precipitated calcium carbonate/cholecalciferol/magnesium carbonate mixture $(2$ tablets/day), rosuvastatin calcium $(2.5 \mathrm{mg} /$ day), aspirin (100 mg/day), a trimethoprim/sulfamethoxazole mixture (1 tablets/day), magnesium oxide (990 mg/day), prednisolone (10 mg/day), and torasemide (4 $\mathrm{mg} /$ day). Lorlatinib was discontinued the day after the onset of visual hallucinations, but both the visual and auditory hallucinations persisted for a further 3 days before disappearing on day 4 after discontinuation. 
Lorlatinib was restarted at $50 \mathrm{mg} /$ day on day 5 after discontinuation, and the visual and auditory hallucinations reappeared the following day. He reported that the visual hallucinations were less powerful and that the auditory hallucinations were about half as loud as before. The specific visual hallucinations included curtains approaching him up close, stuffed animals emerging from the ceiling, and ceiling lights moving. The auditory hallucinations included various pieces of music playing outside the window and above his head. He was diagnosed with grade 1 CNS disorder. There were no findings suspicious for brain metastasis and no medications that could cause delirium. Also, psychiatrists determined that delirium was unlikely because of the lack of cognitive decline and disorientation. Six months later, his general condition deteriorated and lorlatinib was discontinued. However, grade 1 auditory hallucinations persisted, sometimes as singing but mostly instrumental and with familiar and unfamilar pieces. He often experienced these hallucinations in the morning, during quiet times before going to bed, and when it was quiet during the daytime. We show the course of this case in fig.1.

Lorlatinib is expected to be effective against unresectable advanced or recurrent ALK-positive NSCLC that is resistant or intolerant to an ALK tyrosine kinase inhibitor. ${ }^{8-10}$ In our case, there was no organic cause of the visual and auditory hallucinations such as brain damage or brain metastasis and there was no history of psychotic symptoms. Therefore, we suspected the hallucinations to be drug-induced. Auditory hallucinations developed on day 4 and visual hallucinations on day 7 after starting to take lorlatinib $100 \mathrm{mg}$. Given that the half-life of lorlatinib is $20.8 \pm 3.80 \mathrm{~h}$, the blood concentration of lorlatinib theoretically reached a steady state on day 4-5 after the onset of auditory hallucinations. Moreover, the auditory hallucinations disappeared on day 5 after its discontinuation and the visual and auditory hallucinations reappeared after its resumption.

In a study by Bauer, among the CNS disorders by lorlatinib, median time to onset of mood effects was 43 days (range, 1-452 days), that of cognitive effects was 53 days (range, 1-423), speech effects was 42 days (range, 1-404). ${ }^{7}$ However, there were no reports about the time to onset of visual and auditory hallucinations.

We also considered whether concomitant prednisolone may have caused the visual and auditory hallucinations. Prednisolone, a steroid, is administered for drug-induced pneumonitis. In a study on steroid-induced psychiatric syndromes by Lewis and Smith, $43 \%$ of cases developed symptoms during the first week of treatment, $57 \%$ within the first 2 weeks, and $93 \%$ within 6 weeks. ${ }^{11}$ Based on these findings, it would seem unlikely that prednisolone induced the hallucinations in our patient because they developed 2 months after its initiation. In another study, psychiatric symptoms were noted in $1.3 \%$ of 463 patients on prednisolone $40 \mathrm{mg} /$ day, $4.6 \%$ of 175 patients on $41-80 \mathrm{mg} /$ day, and $18.4 \%$ of 38 patients taking $80 \mathrm{mg} /$ day or more. ${ }^{12}$ In the aforementioned study by Lewis and Smith, $23 \%$ of the patients had been receiving less than $40 \mathrm{mg} /$ day of prednisone before the onset of psychiatric symptoms, whereas $77 \%$ had been receiving more than $40 \mathrm{mg} /$ day. ${ }^{11}$ Therefore, the psychological symptoms caused by prednisolone are presumed to be dose-dependent. Psychiatric symptoms were unlikely in the present case because the prednisolone dose at onset of the hallucinations was low $(10 \mathrm{mg} /$ day $)$.

Lorlatinib has been shown to be effective against brain metastasis owing to its high blood-brain barrier penetration. ${ }^{8,9}$ However, the frequency of adverse events in the CNS is reported to be $39.7 \%$. These adverse events include mainly cognitive effects, mood effects, and speech effects ${ }^{7}$, and there are no detailed reports on visual or auditory hallucinations. We suspect that the mechanism behind the visual and auditory hallucinations is related to the effect of lorlatinib on the CNS, but the mechanism remains to be clarified. Because the risk factors associated with adverse events in the CNS are unknown, further study is warranted.

To evaluate the accuracy of the relationship between suspected drug and adverse event, we used the adverse drug reactions probability scale proposed by Naranjo et al. In this case, the hallucinations were classified as "possible" because the score was 7 points (table 1). ${ }^{13}$

In this case, grade 1 auditory hallucinations persisted after lorlatinib was discontinued. Bauer et al. reported that the median time to resolution of CNS disorders by discontinuing lorlatinib was 12.5 days (range 2-112). ${ }^{7}$ However, the hallucinations did not improve in our case, and therefore it is necessary to keep in mind that hallucinations could persist even after discontinuation in patients who develop hallucinations while receiving 
lorlatinib.

What is new and conclusion

We encountered a patient who developed visual and auditory hallucinations caused by lorlatinib. To our knowledge, this is the first detailed report of lorlatinib-induced hallucinations and the persistence of auditory hallucinations after discontinuation. Such scarcity of reports is probably due to the infrequent use of lorlatinib to date.

Conflicts of interest: The authors have no conflicts of interest to declare.

Funding information: None.

Author contributions: JH: contributed to acquisition of data, drafting the manuscript, final revision of the manuscript, and participated sufficiently in the work. HN, HM, KM, HT, SI, KF, and TA: made significant contribution toward editing of the manuscript.

Acknowledgement: The authors thank Sugita of ThinkSCIENCE, Inc. for proofreading our paper.

References

1. Koivunen JP, Mermel C, Zejnullahu K, et al. EML4-ALK fusion gene and efficacy of an ALK kinase inhibitor in lung cancer. Clin Cancer Res 2008;14(13):4275-4283

2. Soda M, Choi YL, Enomoto M, et al. Identification of the transforming EML4-ALK fusion gene in non-small-cell lung cancer. Nature. 2007;448(7153):561-566.

3. Takeuchi K1, Choi YL, Soda M, et al. Multiplex reverse transcription-PCR screening for EML4-ALK fusion transcripts. Clin Cancer Res. 2008;14(20):6618-6624.

4. Gainor JF, Dardaei L, Yoda S, et al. Molecular Mechanisms of Resistance to First- and SecondGeneration ALK Inhibitors in ALK-Rearranged Lung Cancer. Cancer Discov. 2016 ;6(10):1118-1133. Costa DB,

5. Shaw AT, Ou SH, et al. Clinical Experience With Crizotinib in Patients With Advanced ALKRearranged Non-Small-Cell Lung Cancer and Brain Metastases. $\quad$ J Clin Oncol. 2015 10;33(17):18811888.

6. Johnson TW, Richardson PF, Bailey S, et al. Discovery of (10R)-7-amino-12-fluoro-2,10,16-trimethyl15-oxo-10,15,16,17-tetrahydro-2H-8,4-(metheno) pyrazolo[4,3-h] $[2,5,11]$-benzoxadiazacyclotetradecine3 -carbonitrile (PF-06463922), a macrocyclic inhibitor of anaplastic lymphoma kinase (ALK) and c-ros oncogene 1 (ROS1) with preclinical brain exposure and broad-spectrum potency against ALK-resistant mutations. J Med Chem 2014;57(11):4720-4744

7. Bauer TM, Felip E, Solomon BJ, Thurm H, Peltz G, Chioda MD, Shaw AT.Epub 2019 Mar 19. Clinical Management of Adverse Events Associated with Lorlatinib. Oncologist. 24(8):1103-1110.

8. Shaw AT, Felip E, Bauer TM et al. Lorlatinib in non-small-cell lung cancer with ALK or ROS1 rearrangement: An international, multicentre, openlabel, single-arm first-in-man phase 1 trial. Lancet Oncol. 2017;18 (12):1590-1599.

9. Solomon BJ, Besse B, Bauer TM, et al. Lorlatinib in patients with ALK-positive non-small-cell lung cancer: results from a global phase 2 study. Lancet Oncol. 2018;19(12):1654-1667.

10. Lee J, Sun JM, Lee SH, et al. Efficacy and Safety of Lorlatinib in Korean Non-Small-Cell Lung Cancer Patients With ALK or ROS1 Rearrangement Whose Disease Failed to Respond to a Previous Tyrosine Kinase Inhibitor. Clin Lung Cancer. 2019; 20 (3):215-221.

11. Lewis DA, Smith RE. Steroid-induced psychiatric syndromes: A report of 14 cases and a review of the literature. J Affect Disord. 1983; 5 (4);319-332

12. Ismail MF, Lavelle C, Cassidy EM. Steroid-induced mental disorders in cancer patients: a systematic review. Future Oncol. 2017;13(29);2719-2731

13. Naranjo CA, Busto U, Sellers EM, et al. Greenblatt DJ.A method for estimating the probability of adverse drug reactions. Clin Pharmacol Ther. 1981;30(2):239-245.

\section{Hosted file}


fig.1.pptx available at https://authorea.com/users/375107/articles/492472-lorlatinib-inducedvisual-and-auditory-hallucinations-a-case-report 